\title{
Tapping into the potential of remote developmental research: introducing the OxfordBabylab app
}

\author{
Nicola A. Gillen ${ }^{1}$, Serene Siow ${ }^{1}$, Irina Lepădatu ${ }^{1}$, Jelena \\ Sučević ${ }^{1}$, Kim Plunkett ${ }^{1}$, and Mihaela Duta ${ }^{1,}{ }^{*}$ \\ ${ }^{1}$ Department of Experimental Psychology, University of Oxford \\ ${ }^{*}$ Corresponding author: Mihaela Duta, \\ mihaela.duta@psy.ox.ac.uk
}

This is a pre-print of the submission to Front. Psychol. - Developmental Psychology, Research topic: Empirical Research at a Distance: New Methods for Developmental Science. The submission is currently under review.

\begin{abstract}
The widespread use of mobile touchscreen devices in the home provides the opportunity to conduct remote experimental research with toddlers. We present a study aiming to investigate the feasibility and efficacy of using a mobile app for running alternative forced choice tasks with toddlers in the home. The parents of 153 toddlers aged between 12 and 36 months first completed the Oxford Communicative Development Inventory (CDI) as a measure of their child's vocabulary, and then completed a receptive vocabulary task with their child on their own mobile device. We evaluated the feasibility in terms of completion and engagement scores and the efficacy in terms of response systematicity. Our results show that it is both feasible and effective to use parents' own mobile devices to run a study with toddlers in their home. Toddlers were successfully engaged and their responses reflected their integration of the audio-visual context presented. We also introduce our OxfordBabylab app, a cross-platform mobile app which implements a customisable template for alternative forced choice tasks.
\end{abstract}

Keywords: language development research, receptive vocabulary, mobile apps, infant research, alternative forced choice tasks 


\section{Introduction}

A study on touchscreen usage in infants between 5 and 40 months indicated widespread use of touchscreen technology across this age group, especially above 24 months (Cristia and Seidl, 2015). The increasing availability of touchscreen devices offers the opportunity to remotely conduct experimental research in a home setting. This applies in particular to toddler development research, as such studies most often take place in a nursery or school setting or else in a controlled laboratory. Research studies are likely to benefit from taking place in a toddler's home environment, both for the experimenter as well as for the child and their accompanying caregiver. In addition to the convenience of not having to travel to attend a study, children are likely to be more comfortable in their home than in an unfamiliar environment. Running studies via mobile apps on touchscreen devices is likely to be the most convenient way for the general population to participate in research. This would subsequently enable researchers to reach a wider sample of participants than 7 lab-based and other in-person studies are often able to. Participants from a wider range of geographic locations could be reached as well as participants from a wider range of socio-economic statuses.

Experimental paradigms using touchscreen technology require participants to overtly exhibit their response, typically by tapping the device screen. This is in contrast to common experimental procedures used with infants, such as preferential looking tasks, which are frequently conducted with eye-tracking technology and do not require overt responses. Preferential looking tasks have been used to directly measure receptive vocabulary in infants (Killing and Bishop, 2008; Houston-Price et al., 2007; Fernald et al., 1998). In such tasks, all that is required from the infant is to be attentive during the task (that is, to look at the screen presenting the stimuli). When exploring the feasibility of implementing touchscreen tasks, it is therefore important to first determine whether infants can actively engage with a task requiring overt responses, as the success of data collection is fully dependent on this ability.

Tasks using touchscreen technology have been successfully used in research with 3-5-year-old children to measure receptive vocabulary (Golinkoff et al., 2017; Levine et al., 2020) and for the presentation of cross-modal experimental tasks (Lewis et al., 2020). Moreover, touchscreen tasks administered in a laboratory setting have been successfully used with infants as young as 16 months (Friend and Keplinger, 2003). Friend and Keplinger developed the Computerized Comprehension Task (CCT): a touchscreen task to assess infant vocabulary comprehension in an object selection task which requires an overt response from the infant. The CCT was validated with a sample of 16-month-old infants. Infants' attention on the CCT was measured as the number of trials during which which they were looking at the stimuli presented on the touchscreen, recorded manually by the experimenter present. Infants' engagement on the CCT was measured as the number of trials on which they provided an overt response. The CCT was shown to maintain infant attention and engagement significantly better than when the same task with identical stimuli was administered in the more traditional picture-book form. These findings were later replicated in another sample of 16-17 and 20-21-month-old infants with stable test-retest reliability (Friend and Keplinger, 2008). The CCT was optimised for use in a lab 
setting, with verbal prompts towards target words produced by the experimenter (e.g. "Where is the $d o g$ ? Touch dog.") and trial progression was also controlled by the experimenter.

In order to conduct remote experimental research using mobile devices in the home, it is important that a task allows for self-administration (or, in the case of infants, administration by parents). To facilitate this, the mobile apps must include pre-recorded auditory prompts and consist of participant-driven trial progression. The receptive vocabulary task by Golinkoff et al. (2017) (later employed by Levine et al. (2020)) used pre-recorded audio stimuli with 3-5-year-old children. Similarly, Lewis et al. (2020) used pre-recorded stimuli to administer a mutual exclusivity task with 2-5-year-old children. The results of Lewis et al. (2020)'s study suggests that pre-recorded audio stimuli can be successfully used with toddlers as young as 2 years of age with minimal experimenter involvement (N.B. the experimenter provided a short introduction to the task, but the task instructions were given via pre-recorded stimuli presented through the tablet's speakers). Furthermore, Lo et al. (2021) tested the receptive vocabularies of 18-20-month-old Norwegian toddlers under conditions consisting of stimulus pairs with varying levels of semantic relatedness and difficulty (as evaluated by existing normative data). This study was conducted with toddlers completing a mobile app task in a lab or home setting. Results showed that the accuracy and number of responses made did not differ significantly between groups who completed the task in the lab (experimenter-administered) and in the child's home (parent-administered). This particular task, however, is not readily available for use by other researchers.

In addition to pre-recorded stimuli, a mobile app suitable for conducting remote data collection needs to be accompanied by comprehensive instructions so that parents can successfully fulfill the role of the experimenter in guiding their child through the task. The app should also be stored in a format that can be easily accessed by parents online. The mobile app that we present in this paper aims to fill that gap, by providing a customisable template of a visual world paradigm that facilitates convenient remote data collection by infant researchers.

The present study aimed to investigate the feasibility and efficacy of using a mobile app for running alternative forced choice tasks with toddlers in the home, and to introduce the OxfordBabylab app as a customisable tool for running such studies. Our study aimed to answer the following two questions:

1) Do toddlers successfully engage and maintain attention in a cross-modal study run on a mobile device in their own home, in which they give responses by tapping on the screen?

2) Are responses given by toddlers in such a study meaningful, and can they be interpreted to reflect their integration of the cross-modal context?

Families were recruited via emails and social media, and participated in this study entirely from their own home. Parents first completed an online questionnaire on their child's vocabulary (Hamilton et al., 2000), then downloaded the OxfordBabylab mobile app (from Apple Store or Play Store) on their own device and completed an alternative forced choice receptive vocabulary task with their child.

We predicted that the majority of toddlers would be able to engage successfully with our mobile 
app and maintain attention enough to provide a high number of valid trials. We also predicted that the toddlers who showed engagement with the app would also respond systematically and that the accuracy of their responses would be predicted by parental reports of word comprehension. If met, these predictions would demonstrate the feasibility and efficacy of using the OxfordBabylab mobile app for developmental research with toddlers.

\section{Materials and Methods}

\subsection{Procedure}

Participants were recruited with advertisements which were posted on Facebook or emailed to parents who were signed up to a lab research database. Parents who replied to express their interest in the study were sent detailed information forms. Parents who agreed to participate in the study submitted informed, online consent. Participation in the study comprised of the following two steps, which were completed within two weeks of each other:

Oxford CDI - parents completed an online questionnaire on their child's vocabulary, see Section 2.3

OxfordBabylab app - parents guided their child to complete a forced choice receptive vocabulary task in the form of a mobile app on the parent's own mobile touchscreen device, see Section 2.4

\subsection{Participants}

Our sample comprised of 153 monolingual toddlers learning British-English in the UK, ranging in age from 12 to 36 months. For data visualization and analysis, the sample was split into four age groups:

12 - 19: $16.23 \pm 1.95$ months, $N=22,7$ females

20 - 24: $22.26 \pm 1.17$ months, $N=50,22$ females

25 - 29: $26.38 \pm 1.25$ months, $N=39,16$ females

30 - 36: $33.07 \pm 2.09$ months, $N=42,19$ females

No toddlers were reported to have any ongoing hearing issues at the time of participation.

\subsection{Oxford Communicative Development Inventory}

The Oxford Communicative Development Inventory (Oxford CDI, Hamilton et al. $(2000)$ ) is a tool for gathering information about infant and toddler receptive and productive vocabulary through parental reports. The Oxford CDI contains 418 English words commonly known to infants learning British-English grouped into 19 categories. Of these, 232 words are nouns, split across 10 semantic categories. 
For each word in the Oxford CDI, parents are asked to indicate whether their child: 1) understands the word, 2) understands and says the word, or 3) does not know the word. This allows the calculation of a comprehension score for each child using the number of words that the child either 1) understands or 2) understands and says, as reported by their parent. Parents completed the Oxford CDI in the form of an online questionnaire. Word categories were presented in random order, while items within a category were presented in alphabetical order.

\subsection{The OxfordBabylab mobile app}

The OxfordBabylab mobile app implements a customizable template for running an alternative forced choice task on the user's own Android and iOS devices (phone or tablet) and is available to download from both Google Play Store (Duta, 2020b) and Apple App Store (Duta, 2020a).

The OxfordBabylab app is comprised of three sections: practice, study and play. The sections are identical in structure and take the form of a game in which a fictional character Flora the Flower guides through a series of trials using audio narratives with engaging toddler-friendly language.

Each section begins with a short animation of Flora the Flower introducing herself and explaining the game. A visual timeline of a trial is illustrated in Figure 1. Trials are started manually by tapping on a loop animation of Flora the Flower. Each trial starts with an audio prompt followed by the presentation of a pair of pictures for a maximum of 10 seconds. During this time a response is expected in the form of a tap on one of the pictures as instructed in the audio prompt. If the response given is wrong, the picture for the correct response looms in-and-out as a visual reminder of the game's goal and the trial is repeated once starting with the loop animation. If no response is provided, the trial times out and is also repeated once. If the correct response is given, the tapped picture is briefly replaced by Flora the Flower as a visual reward along with a ding sound and the sequence advances to the next trial.

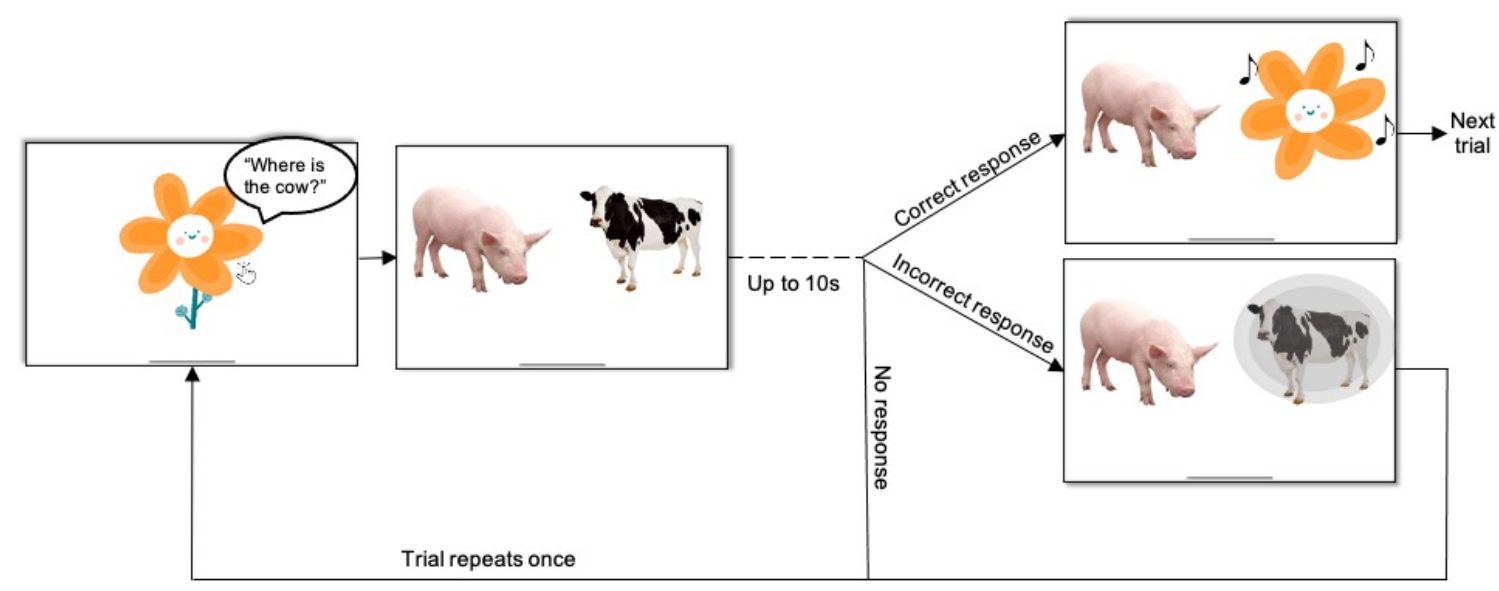

Figure 1: The timeline of each trial, showing feedback presented for correct and incorrect responses, as well as trial repetition for incorrect responses and no responses. A video showing the practice section is available at: https://tinyurl.com/4r9t55f5

The identity of the audio and visual stimuli in each trial, as well as the trial order, are set at the 
time of running the app via an activation code that unlocks and tailors the functionality of the app. The activation code is unique, single-use and fully anonymised, and is provided to each parent by the research team upon completion of the Oxford CDI. A cloud database contains specifications for a variety of study set-ups and the activation code directs the app to retrieve one of these set-ups. This allows researchers to personalise the trials to individual participants, if required $!^{1}$

The unlocked app first prompts parents to agree to participate and then allows them to choose between running the practice or the study. Parents are encouraged to first complete the practice on their own and then with their child, before proceeding to the study with their child. Parents are instructed during the practice to encourage their child to tap the screen themselves. If the child is reluctant to do so and prefers instead to simply point to the image of their choice, parents are instructed to tap on their behalf making sure to follow exactly the child's choice, irrespective of its accuracy.

The response data from the study is uploaded to a cloud database: either at the completion of the study, or earlier if parents decide to prematurely end the study. If the parents decide to not complete the study, they are offered the choice of whether or not to upload partial response data. If the study is completed, the response data is automatically uploaded. All data is deleted from the local device at the completion of each section. Responses from the practice and play sections are never uploaded.

The activation code also provides the means for the linkage of any uploaded study data with the participant's Oxford CDI comprehension score and demographic information. The linkage between the activation code and the participant is done offline and is completely separate from the cloud database that receives the uploaded response data from the mobile app.

Once the study section is run, either completely or partially, the app locks the practice and study sections and unlocks the play section, which remains active in the app for as long as the app remains installed on the device. The play section is automatically activated after an attempt to the study section, irrespective of whether the study was completed or data was uploaded.

\subsection{The task}

For the purpose of this study, the OxfordBabylab app was set up to implement a two-picture alternative forced choice receptive vocabulary task. Flora the Flower asked toddlers to help her 'find' objects by tapping on one of the two presented pictures. Each trial showed a target (the required object) and a distractor (a different object that is not named) shown in a left-right arrangement. The study section comprised of 32 trials, the practice section of 4 trials and the play section of 10 trials.

\footnotetext{
${ }^{1}$ Please note that currently the OxfordBabylab app is only available for download from the UK. If download is required from outside the UK please contact the corresponding author.
} 


\subsection{Stimuli}

The items of the study section were 64 nouns selected from the Oxford CDI list. Items were selected on the basis that they could be visually presented as unambiguous objects which could be labelled as the corresponding word in the CDI by both adults and young children.

The sixty-four study items were divided into 32 target and 32 distractor item sets. Within each set, half of the items were selected, based on existing Oxford CDI normative data (Hamilton et al. 2000), as being less likely to be known by 21-month-old children (15\%-50\% of normative sample) and the other half were selected as being more likely to be known by 21-month-old children (94\%-98\%) based on the same normative data. This selection was made to minimise the likelihood of ceiling effects in toddlers' performance. Items in each pair were also chosen either from the same or a similar semantic category where possible, and the visual appeal of their illustrations was matched. The study trials were presented in a fixed quasi-random order, counterbalancing the position of the target (see Supplementary Material).

The 8 items for the 4 practice trials and the 20 items for the 10 play trials were from an extended version of the Oxford CDI list and chosen to be different from the study items.

Visual stimuli for study trials consisted of royalty-free illustrations of the items sourced from free, online databases. Visual stimuli for the practice and play trials were cartoon images selected from similar sources.

Audio stimuli were labels for the target pictures embedded in the carrier phrase "Where is the $X "$ and were generated via an online Text-to-Speech synthesiser (Acapela-Box, Acapela Group 2020). All generated audio files resembled natural speech in a female child's voice with a British-English accent. Audio files with the same generated voice were used to provide the introduction and a goodbye message.

The fictional character Flora the Flower was represented by a loop GIF animation of a cartoon flower ${ }^{2}$

\subsection{Measures and analysis}

We considered a response in a trial to be valid if the response latency was greater than 0.35 seconds. This threshold was chosen based on existing evidence of adults' typical tapping times to visual stimuli in a touchscreen gaming task (Lee et al., 2015). Response accuracy was measured for the first tap made by participants during either the initial or the repeat presentation of the visual stimuli. For each trial with a valid response (valid trial), the response accuracy falls into one of the following two categories:

correct response: child tapped the target item

incorrect response: child tapped the distractor item

Trials where no response was given are not considered in the evaluation of a trial accuracy

\footnotetext{
${ }^{2}$ Attribution for GIF: https://www.instagram.com/orlandokorzo/
} 
because there could be several reasons why a child does not respond. Lack of knowledge of the presented items is one of the potential reasons leading to a lack of response in a trial, but another could be that the child did not attend to the trial. The response accuracy score for each child was the percentage of valid trials with correct responses.

We defined the following two feasibility measures:

Completion score is the percentage of uploaded study trials and is used to evaluate parents' administration of the study. We interpret a $100 \%$ completion score to mean that parents successfully completed the study with their child, and a low completion score as an indication that parents encountered challenges in completing the study (e.g. because their child lost interest or was not cooperative).

Engagement score is the percentage of valid trials (Lo et al., 2021; Friend and Keplinger, 2003).

Using these measures we split the participants as follows:

low completion - completion score of under 28

low engagement - engagement score of under 28

high completion and engagement - both completion and engagement score of at least 28

All statistical analyses were performed only on high completion and engagement participants. The threshold of 28 trials was selected to ensure sufficient counterbalancing in the trials for the participants entered into the analyses, see Section 2.6.

We studied response systematicity using the response accuracy, both on its own and in terms of its relationship with the reported knowledge of the trial items. For each trial, parental report of child knowledge of the trial items falls into one of the following four categories:

both-known: child reported to know both the target and distractor items

target-known: child reported to only know the target item

distractor-known: child reported to only know the distractor item

neither-known: child reported to not know the target or the distractor item

We conducted one-sample t-tests on response accuracy scores for each age group in order to investigate whether participants were responding significantly above chance and not at random (50\%). We predicted that if participants were successfully engaging with the app, as indicated by their high completion and engagement scores, then their response accuracy scores would be significantly greater than chance. We investigated the effect of age on response accuracy scores by way of a one-way ANOVA with age group as an independent variable. We predicted that response accuracy scores would increase with age.

We also conducted a binomial mixed-effects analysis to investigate whether response accuracy was predicted by parental report of knowledge of individual trial items. We expected that if children were responding systematically, they would be most likely to respond correctly for target-known and both-known trials, and at chance for neither-known trials. For distractorknown trials, we will consider in the discussion the possibility that toddlers may use mutual exclusivity to give a correct response (Markman et al., 2003). 


\section{Results}

\subsection{Completion and engagement scores}

Figure $2 \mathrm{a}$ gives the distribution of participants across the three completion and engagement groups. The figure shows that more than $50 \%$ of the participants in the 12-19 months age group are either in the low completion or in the low engagement group (either one excludes the participant from analysis). For the older age groups, the majority of the participants completed and engaged in a high number of trials.

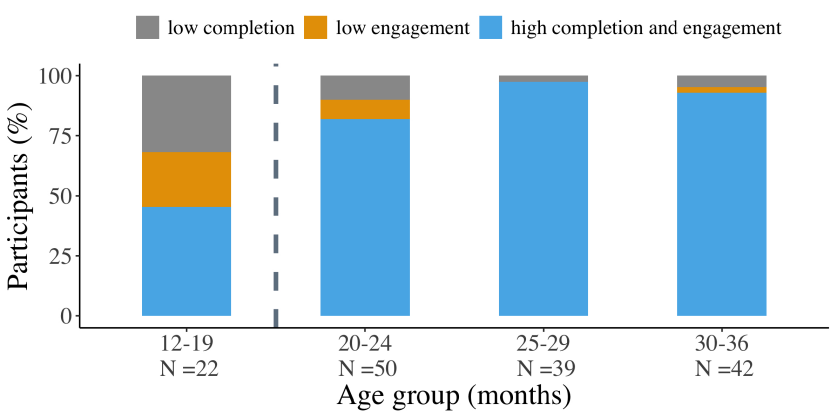

(a)

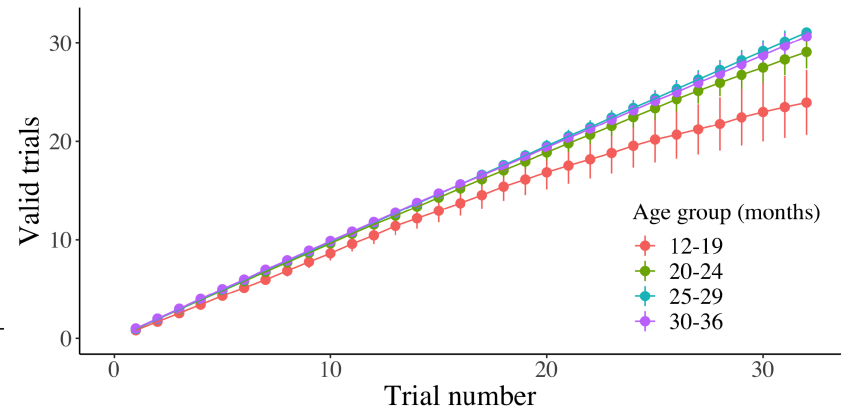

(b)

Figure 2: (a) Distribution of participants across the completion and engagement groups. (b) Timecourse for the cumulative sum of valid responses across trials. Bars: $95 \%$ confidence intervals.

Figure 2b gives the timecourse for the cumulative sum of valid trials. The figure shows that the four age groups behave similarly until approximately midway through the study; in the second half of the study a clear trend of fewer valid responses is observed from the youngest age group. Analyses were conducted only on the participants who showed high completion and engagement ( $N=128:$ 12-19 months $N=10,20-24$ months $N=41,25-29$ months $N=38,30-36$ months $N=39$ ). As there were only 10 participants in the 12-19 months age group, this group was excluded from comparative statistical analyses with the other three age groups.

Figure 3 illustrates the response times for high completion and engagement participants. Response times decreased with age, showing that children were quicker to make their first tap the older they were.

\subsection{Response systematicity}

We evaluated response systematicity in terms of the response accuracy on its own, and also in terms of its relationship with the item comprehension status as reported by parents. 


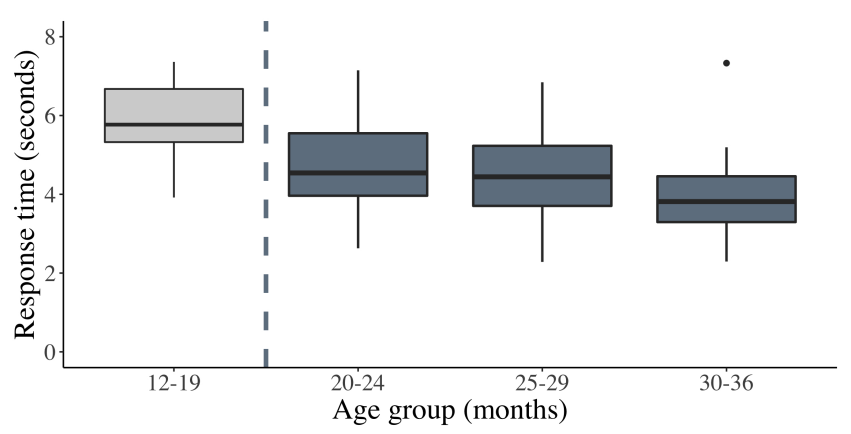

Figure 3: Response times relative to visual stimuli onset for high completion and engagement participants. Hinges: upper and lower quartiles.

\subsubsection{Response accuracy score}

Figure $4 a$ presents the response accuracy for each age group, showing that accuracy increases with age. A one-way ANOVA revealed that this was a significant increase $[F(2,115)=11.15, p<$ $\left..001, \eta^{2}=0.19\right]$. The largest difference in scores was between the 20-24 months and 30-36 months groups $[M: 8.86 \%, p<.001]$. The difference between the scores of the $25-29$ months and 30-36 months groups was also statistically significant $[M: 5.23 \%, p=.02]$. The difference between the 20-24 months and 25-29 months groups was not significant [ $M: 3.63 \%, p=.14]$.

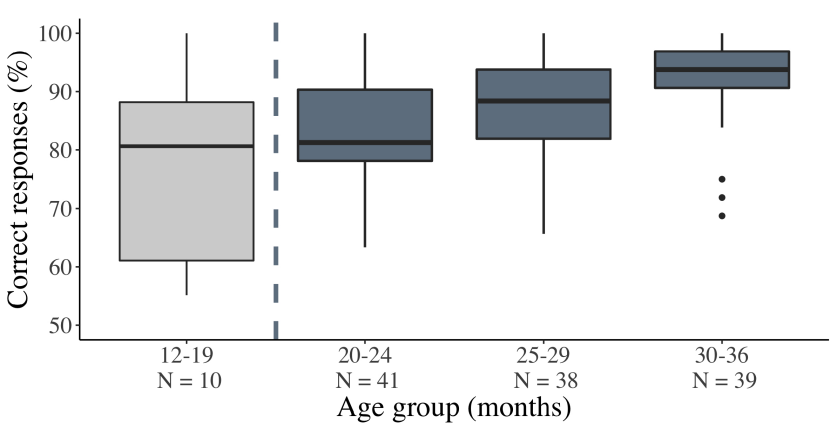

(a)

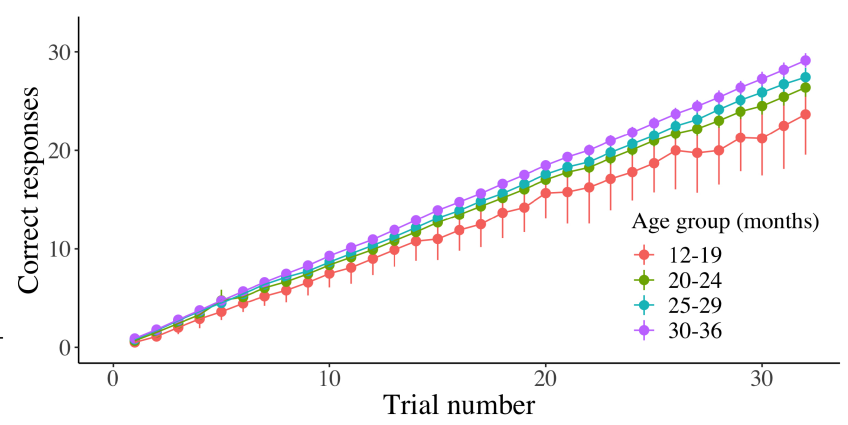

(b)

Figure 4: (a) Distribution of correct responses across age groups. Hinges: upper and lower quartiles. (b) Timecourse for the cumulative sum of correct responses across trials. Bars: 95\% confidence intervals.

Table 1 gives the results of the one-sample t-tests. These revealed that response accuracy scores were significantly greater than chance for all age groups:

\begin{tabular}{ccrrrcr} 
Age group & $M(\%)$ & \multicolumn{1}{c}{$s t d$} & \multicolumn{1}{c}{$d f$} & \multicolumn{1}{c}{$t$} & Cohen's $d$ & \\
$\mathbf{1 2}-\mathbf{1 9}$ & 76.86 & 16.35 & 9 & 5.20 & 1.64 & $* * *$ \\
$\mathbf{2 0}-\mathbf{2 4}$ & 83.02 & 9.04 & 40 & 23.39 & 3.65 & $* * *$ \\
$\mathbf{2 5}-\mathbf{2 9}$ & 86.65 & 8.81 & 37 & 25.65 & 4.16 & $* * *$ \\
$\mathbf{3 0}-\mathbf{3 6}$ & 91.88 & 7.27 & 38 & 35.95 & 5.76 & ${ }^{* * *}$ \\
\hline${ }^{* * *} p<.001$ & & & & & &
\end{tabular}

Table 1: Statistical results of the one-sample t-tests on response accuracy

Figure $4 \mathrm{~b}$ gives the timecourse for the cumulative sum of accurate responses. The figure shows 
that the differences in accuracy across age groups remained constant for all trials for all but the youngest 12-19 months age group, whose timecourse deviates approximately midway through the study.

\subsubsection{Response accuracy and item comprehension status}

Table 2 shows the results of the binomial mixed effects model to which parental report of trial item knowledge and age group were submitted as fixed effects, while participant and trial were random effects, as justified by Akaike's Information Criterion (Akaike, 1974). Figure 5 a illustrates the predicted marginal means of trial response accuracy across age groups and reported item knowledge. Figure 5b gives the average number of trials for each category of item knowledge. The figure shows that on average, across all age groups, participants had considerably greater both-known trials and there were proportionately fewer target-known, distractor-known and neither-known trials.

Main effects were derived from Chi-Square tests and follow-up comparisons were conducted with Tukey tests. There was a significant main effect of the reported item knowledge on trial response accuracy. Participants were significantly more accurate on both-known trials $[M=21.82 \pm 3.99$ trials $]$ compared to distractor-known $[M=2.78 \pm 0.33$ trials $]$ and neitherknown trials $[M=4.26 \pm 1.80$ trials]. There was no significant difference in response accuracy between both-known and target-known trials $[M=3.30 \pm 0.34$ trials]. There was also no significant difference in accuracy between distractor-known and neither-known trials.

\begin{tabular}{|c|c|c|c|c|c|c|c|c|c|c|}
\hline Main Effects & $\chi^{2}$ & $d f$ & & \multicolumn{3}{|c|}{ Comparisons } & $\beta$ & S.E. & $z$ & \\
\hline Item knowledge & 28.77 & 3 & $* * *$ & $\begin{array}{l}\text { both } \\
\text { both } \\
\text { both } \\
\text { neither } \\
\text { target } \\
\text { target }\end{array}$ & $\begin{array}{l}- \\
- \\
- \\
- \\
-\end{array}$ & $\begin{array}{l}\text { distractor } \\
\text { neither } \\
\text { target } \\
\text { distractor } \\
\text { distractor } \\
\text { neither }\end{array}$ & $\begin{array}{r}-\mathbf{0 . 6 1} \\
-0.46 \\
0.13 \\
0.14 \\
\mathbf{0 . 7 4} \\
0.59\end{array}$ & $\begin{array}{l}\mathbf{0 . 1 8} \\
0.19 \\
0.22 \\
0.21 \\
\mathbf{0 . 2 5} \\
0.23\end{array}$ & $\begin{array}{r}-3.38 \\
-2.45 \\
0.59 \\
0.68 \\
\mathbf{2 . 9 9} \\
2.55\end{array}$ & $* *$ \\
\hline Age group & 14.35 & 2 & $* * *$ & $\begin{array}{l}20-24 \\
\mathbf{2 0}-\mathbf{2 4} \\
\mathbf{2 5}-\mathbf{2 9}\end{array}$ & & $\begin{array}{l}25-29 \\
\mathbf{3 0 - 3 6} \\
\mathbf{3 0}-\mathbf{3 6}\end{array}$ & $\begin{array}{l}0.26 \\
\mathbf{0 . 8 3} \\
\mathbf{0 . 5 6}\end{array}$ & $\begin{array}{l}0.18 \\
0.20 \\
0.20\end{array}$ & $\begin{array}{l}1.47 \\
4.19 \\
2.81\end{array}$ & $\begin{array}{l}* * * \\
* *\end{array}$ \\
\hline
\end{tabular}

${ }^{* * *} p<.001,{ }^{* *} p<.01,{ }^{*} p<.05$

Table 2: Statistical results of the binomial mixed-effects model

A significant main effect of age group was also observed on trial response accuracy. The 30-36 months age group was significantly more accurate $[M=29.13 \pm 2.24$ trials $]$ than the $20-24$ months age group $[M=26.01 \pm 2.88$ trials $]$, and the $25-29$ months age group $[M=27.43 \pm 2.75$ trials]. No other directions of difference in accuracy between the age groups were statistically significant. 


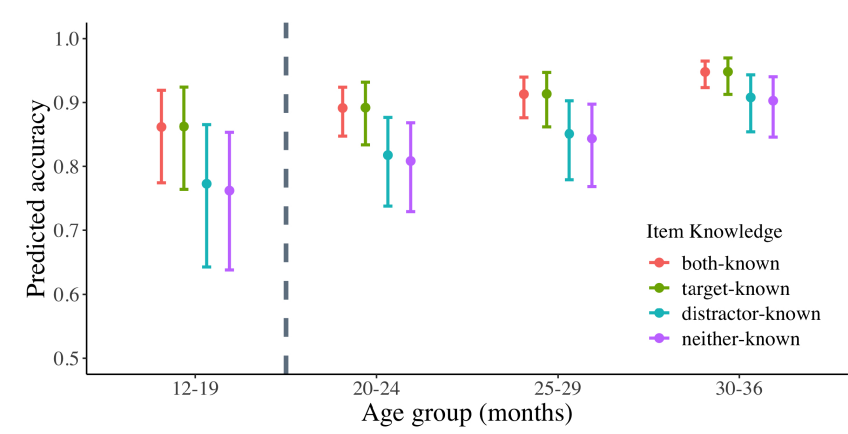

(a)

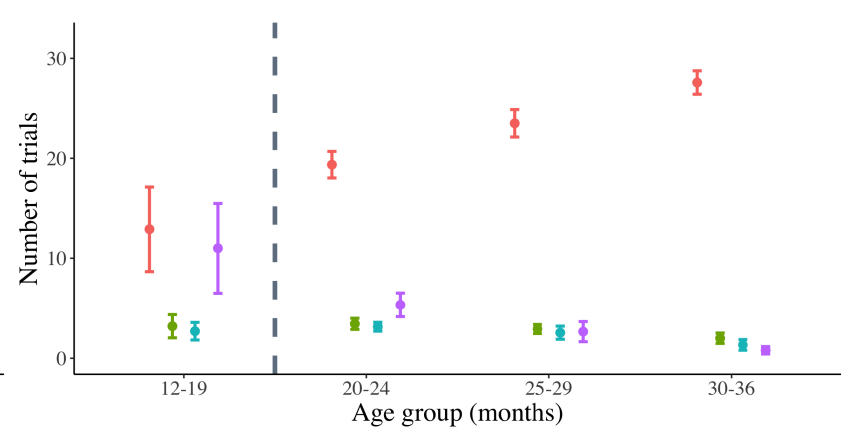

(b)

Figure 5: (a) The predicted probabilities of participants' trial response accuracy. Bars: 95\% confidence intervals. Note that y-axis begins at 0.5 (chance). (b) The number of trials across item knowledge conditions. Bars: $95 \%$ confidence intervals.

\section{Discussion}

The aim of this study was to investigate the feasibility and efficacy of using a mobile app for a receptive vocabulary task performed by toddlers on their parents' own device. We operationalised feasibility in terms of completion and engagement measures and efficacy in terms of response systematicity.

\subsection{Feasibility: completion and engagement}

The vast majority of toddlers successfully completed at least 28 trials. The only exception was the 12-19 months age group, which were found to have both low completion and low engagement scores in comparison to the three older groups. Timecourses for the cumulative sum of the number of valid trials across the study revealed that the 12-19 months group start to deviate from the behaviour of the three older groups approximately midway through the study. This indicates that the younger toddlers were not able to maintain attention and disengaged, suggesting that a shorter task might be more appropriate for them.

Comments we received from parents have shed light on some of the possible reasons for the high attrition rate of younger toddlers by suggesting that some may not have had adequate dexterity for their taps to consistently be recorded by the device as responses. This is consistent with observations from previous studies with this age group, for example Cristia and Seidl (2015) who documented that toddlers throughout the second year frequently perform actions such as banging on touchscreens with an open hand, pressing on the touchscreen for an extended period of time, as well as quickly brushing the surface of the touchscreen with their fingertip. Such actions do not typically bring about desired results when interacting with touchscreens.

While we were unable to statistically compare our 12-19 months group with the older age groups, a recent study showed that toddlers aged 18-20 months were capable of completing a touchscreen two alternative forced choice receptive vocabulary task (Lo et al., 2021). Our 12-19 months group is, however, comparable with this sample in terms of their engagement 
(defined in Lo et al. (2021) as 'attempted trials'). Our 12-19 months group completed on average $M=93.43 \% \pm 4.02$ valid trials, and Lo et al.'s sample 'attempted' on average $91.67 \%$ trials. We surmise that the greater attrition rate we observed is primarily due to the different way of processing the trials with no response in our study. We excluded such trials from analyses as invalid trials (see Section 2.7 for more details) while Lo et al. coded them as synonymous to trials with an incorrect response. Another possible reason is that Lo et al. 's study was run on large tablet devices, whereas in our study we did not control or have information about the type and size of the device used, which could be anything from a small phone to a large tablet. It is possible that younger toddlers find it more difficult to get engaged or interact with phones or smaller tablets.

The high completion and engagement we observed in the three older age groups suggest that overall toddlers are able to interact with the task delivered on a mobile device. The delivery of the task as a game likely helped maintain toddlers' attention and completing the task in their home with their parent was likely to be more comfortable than in an unfamiliar environment. Another benefit of the design of our study was that parents could choose a time that was most suitable for them and their child to complete the task, whereas lab-based studies often do not allow this luxury. We note that while the increase in engagement scores likely stems from the developmental change in toddlers' sustained attention capacities Choudhury and Gorman, 2000), this increase may also be brought about by older toddlers' relatively larger experience, and subsequently expertise, in interacting with touchscreen devices.

\subsection{Efficacy: response systematicity}

Overall the toddlers in our study have accuracy scores well above chance across all age groups, clearly indicating that their tap responses were not random. Our results also support our prediction that the toddlers respond in a meaningful way, reflecting their integration of the cross-modal context. Furthermore, our results also show that toddlers' response accuracy for selecting a target item was based on their vocabulary knowledge.

As predicted, on trials where toddlers were reported to know the target item, they were significantly more accurate as compared to on trials where they were not reported to know the target. This is in line with previous studies that show parental CDI reports for words which toddlers know can accurately predict their responses to target items in tasks (e.g., Killing and Bishop (2008)).

Toddlers in our study also responded well above chance on trials where their parent reported that they did not know the target word. There could be several possible explanations for this. One is that toddlers could have used the mutual exclusivity strategy to obtain a correct response in the trials where they did not know the target but did know the distractor. In those trials they could infer the correct response through exclusion (Markman et al., 2003). If toddlers in this study were using mutual exclusivity, we would expect response accuracy on distractor-known trials to be equal to both-known and target-known trials. However, across all age groups, 
accuracy on distractor-known trials was lower than for the other two aforementioned types of trials (significantly lower among the three oldest age groups).

Another possibility is that parental CDI responses may underestimate toddlers' vocabulary knowledge. Previous studies with toddlers under 24 months have similarly suggested that the CDI underestimated the toddlers' receptive vocabulary knowledge as demonstrated by their behaviour in a preferential looking, eye-tracking task (Houston-Price et al., 2007). Receptive vocabulary is arguably a difficult capacity to measure from parental report, especially during the toddler years which are a period of rapid language growth (Goldfield and Reznick, 1990). Parents may find it challenging to accurately report words they believe their child to understand as opposed to reporting words which they have heard them produce. This may result in parents showing a tendency to be more conservative in their responses, which highlights the importance of also having a direct child-response measure of receptive vocabulary.

\section{Conclusion}

In this paper, we introduced our OxfordBabylab app, a cross-platform mobile app available for download from Google and Apple Store, which implements a customisable template for alternative forced choice tasks 3 . In our study we set up the OxfordBabylab app to implement a receptive vocabulary task, but the app can be also set up to run any audio-visual alternative forced choice task.

Using mobile technology to run developmental studies opens new opportunities to reach wider samples of participants than is typically possible for lab-based studies. We show that it is both feasible and effective to use parents' own mobile devices to run a cross-modal alternative forced choice task with toddlers in their home. Toddlers were successfully engaged and their responses as taps on the screen reflected their integration of the audio-visual context presented.

\section{Conflict of Interest Statement}

The authors declare that the research was conducted in the absence of any commercial or financial relationships that could be construed as a potential conflict of interest.

\section{Author Contributions}

NG - methodological design of the task, stimuli sourcing and generation, data collection, processing and analysis, and write-up.

SS - data processing and analysis, and write-up.

\footnotetext{
${ }^{3}$ If you are interested in using or adapting the OxfordBabylab app please get in touch with the corresponding author.
} 
IL - methodological design of the task, stimuli sourcing and generation, data collection and processing, and write-up.

JS - data analysis and write-up.

$\mathrm{KP}$ - project conception, data analysis and write-up.

MD - project conception and supervision, methodological design of the task, mobile app design and implementation, data analysis, and write-up.

\section{Funding}

This work was prompted during the first UK national lockdown during the COVID-19 pandemic. This work had no allocated funding and was conducted outside of the main grants from which the authors are funded. During this work, NG, SS and IL were funded by a Economic and Social Research Council grant CQR01830 to KP. JS and MD were funded by a Leverhulme Trust grant RPG-2017-307 to KP. MD was also funded by a OUP John Fell Fund grant to Gaia Scerif.

\section{Acknowledgments}

We would like to thank the parents who participated in this study with their toddlers.

\section{References}

H. Akaike. A new look at the statistical model identification. IEEE transactions on automatic control, 19(6):716-723, 1974.

N. Choudhury and K. S. Gorman. The relationship between sustained attention and cognitive performance in 17-24-month old toddlers. Infant and Child Development, 9(3):127-146, 2000.

A. Cristia and A. Seidl. Parental reports on touch screen use in early childhood. PLoS One, 10 (6):e0128338, 2015.

M. Duta. OxfordBabylab iOS app. apps.apple.com/gb/app/oxford-babylab/id1514765773, 2020a.

M. Duta. OxfordBabylab Android app. play.google.com/store/apps/details?id=uk.ac. ox.oxfordbabylab, 2020b.

A. Fernald, J. P. Pinto, D. Swingley, A. Weinberg, and G. W. McRoberts. Rapid gains in speed of verbal processing by infants in the 2nd year. Psychological Science, 9(3):228-231, 1998.

M. Friend and M. Keplinger. An infant-based assessment of early lexicon acquisition. Behavior Research Methods, Instruments, \& Computers, 35(2):302-309, 2003. 
M. Friend and M. Keplinger. Reliability and validity of the computerized comprehension task (cct): data from american english and mexican spanish infants. Journal of Child Language, $35(1): 77,2008$.

B. A. Goldfield and J. S. Reznick. Early lexical acquisition: Rate, content, and the vocabulary spurt. Journal of Child Language, 17(1):171-183, 1990.

R. M. Golinkoff, J. G. De Villiers, K. Hirsh-Pasek, A. Iglesias, M. S. Wilson, G. Morini, and N. Brezack. User's Manual for the Quick Interactive Language Screener (QUILS): A Measure of Vocabulary, Syntax, and Language Acquisition Skills in Young Children. Paul H. Brookes Publishing Company, 2017.

A. Hamilton, K. Plunkett, and G. Schafer. Infant vocabulary development assessed with a british communicative development inventory. Journal of Child Language, 27(3):689-705, 2000.

C. Houston-Price, E. Mather, and E. Sakkalou. Discrepancy between parental reports of infants' receptive vocabulary and infants' behaviour in a preferential looking task. Journal of Child Language, 34(4):701-724, 2007.

S. E. Killing and D. V. Bishop. Move it! visual feedback enhances validity of preferential looking as a measure of individual differences in vocabulary in toddlers. Developmental Science, 11 (4):525-530, 2008.

A. Lee, K. Song, H. B. Ryu, J. Kim, and G. Kwon. Fingerstroke time estimates for touchscreenbased mobile gaming interaction. Human Movement Science, 44:211-224, 2015.

D. Levine, A. Pace, R. Luo, K. Hirsh-Pasek, R. M. Golinkoff, J. de Villiers, A. Iglesias, and M. S. Wilson. Evaluating socioeconomic gaps in preschoolers' vocabulary, syntax and language process skills with the quick interactive language screener (quils). Early Childhood Research Quarterly, 50:114-128, 2020.

M. Lewis, V. Cristiano, B. M. Lake, T. Kwan, and M. C. Frank. The role of developmental change and linguistic experience in the mutual exclusivity effect. Cognition, 198:104191, 2020.

C. H. Lo, A. Rosslund, J. H. Chai, J. Mayor, and N. Kartushina. Tablet assessment of word comprehension reveals coarse word representations in 18-20-month-old toddlers. Infancy, n/a (n/a), 2021. doi: https://doi.org/10.1111/infa.12401.

E. M. Markman, J. L. Wasow, and M. B. Hansen. Use of the mutual exclusivity assumption by young word learners. Cognitive Psychology, 47(3):241-275, 2003. 